\title{
Longitudinal Growth of High Socioeconomic Status Guatemalan Children Analyzed by the Preece-Baines Function: An International Comparison
}

\author{
BARRY BOGIN ${ }^{1}$, MAUREEN WALL ${ }^{1}$, AND ROBERT B. MACVEAN ${ }^{2}$ \\ ${ }^{1}$ Department of Behavioral Sciences, University of Michigan, Dearborn, \\ Michigan 48128; '2Universidad del Valle de Guatemala, Apartado Postal \\ No. 82, Guatemala City, Guatemala
}

\begin{abstract}
The Preece-Baines model I function, adapted for use with a personal computer, is applied to the longitudinal growth records of Guatemalan children and adolescents of high socioeconomic status. The fit of the Preece-Baines function to the Guatemalan data is compared with those of published analyses of the function fitted to the growth of British, Belgian, urban and rural Indian, Australian Aborigine, and African children. Guatemalan, British, and Belgian samples share generally favorable environments for human development and show few differences in the amount and velocity of growth, or in the timing of growth events. Urban Indians live under relatively good environmental conditions and are similar to Guatemalans in the timing of growth events, but grow more slowly and grow less than the Guatemalans, British, or Belgians. Rural Indian, Australian, and African samples live in environments that delay or retard growth, and these last-named three samples grow more slowly, delay the onset of the adolescent growth spurt, and achieve smaller adult height than the Guatemalans. Parameters of the Preece-Baines model are compared between all samples and show that there are several alternate paths in the rate of growth and the timing of adolescent growth events that may be taken from childhood to adulthood.
\end{abstract}

During human childhood and adolescence the shape of the curve of growth of normal individuals follows a predictable course, both in terms of amount and rate of growth. This regularity allows for the mathematical analysis of the distance and velocity curves of growth. By fitting mathematical models, variation between individuals or groups may be quantified and the cause of such variations may be investigated. In this paper, longitudinal records of growth in height for a sample of Guatemalan children are analyzed using the Preece-Baines model I function (Preece and Baines, 1978). The PreeceBaines function is fitted to the longitudinal data using a non-linear least-squares technique. The model contains five mathematical parameters. The function is particularly useful for analyzing adolescent growth since four of the parameters correlate with amount or rate of growth at the onset of the adolescent growth spurt or during the spurt. The fifth parameter correlates with adult height. Mathematical and biological parameters derived from this analysis are compared with published values of the same parameters from European, African, Indian, and Australian children. Variation in growth between samples is discussed in relation to nutrition, health, and standard of living of each population.

\section{MATERIALS AND METHODS Subjects}

The Guatemalan sample is derived from the Longitudinal Study of Child and Adolescent Development conducted by the Universidad del Valle de Guatemala. The individuals selected for the present analysis were students attending a private fee-paying school, one of the most expensive in the country. The school population includes Guatemalan, European, and North American nationals. The sample selected from this school population was limited to Guatemalan nationals whose parents and grandparents have Spanish surnames and high socioeconomic status (SES; defined by parental occupation and education). This de-

Received December 28, 1988; accepted January 22, 1990 
fines a Guatemalan, Hispanic ethnic group. Both school and home environments ensure that these children are well nourished and healthy (Johnston et al., 1973; Bogin and MacVean, 1982, 1983).

A total of 86 boys and 96 girls are included in the sample. Their heights were measured, to the nearest half-centimeter, once a year, normally during the same week every August. Each child had at least 8 annual measurements, but most had 9 or 10 . The absolute age range for the sample from first to last measurement is 5.0 to 17.99 years. Measurement procedures were described by Bogin and MacVean (1978) and generally follow the methods of Cameron (1986). Several technicians made the measurements. Intra-observer reliability coefficients are greater than 0.90 , while inter-observer reliability coefficients average 0.74 . No systematic trends in the error correlations are apparent.

The other samples used for comparison with the Guatemalan children are described briefly here. More detail about each may be found in the original sources. British children are those described by Preece and Baines (1978) in their original presentation of the Preece-Baines model. Exact ages of this sample of 35 boys and 23 girls were not given; rather, Preece and Baines describe the sample as children who "... had grown less than $1 \mathrm{~cm}$ during the last year of measurement, and had at least two years of measurements available before the onset of puberty."

The Belgian sample of 50 girls and 48 boys were measured once a year, on or about their birthdays, between the ages of 2.0 and 18.0 years of age. They are part of a nationally representative sample of healthy, well-nourished children (Hauspie et al., 1980a; Wachholder and Hauspie, 1986; Hauspie and Wachholder, 1986). The Preece-Baines parameters used in the present analysis represent the 50th centile values for this sample and were kindly supplied by Dr. Hauspie.

The urban Indian sample is of middleclass Bengali children-63 boys and 42 girls. The children were ". . . measured at regular intervals over periods of up to 14 years," including the period from the beginning of the adolescent spurt to the cessation of growth. These middle-class Bengalis were taller than the median values of Indian growth standards, suggesting that they lived under better than average environmental conditions for India. However, these urban
Indians grew, on average, between the 5th and 10th percentile of British standards (Hauspie et al., 1980b).

The rural Indian sample includes 47 boys from the Hyderabad region who were diagnosed as suffering from "severe childhood undernutrition"; their heights at age 5 years averaged 4.0 standard deviations below the Harvard reference values for well-nourished children. Between 13 to 16 height measurements, starting at age 5.0 years, are available for each boy (Satyanarayana et al., 1989).

The Australian sample consists of $62 \mathrm{Ab}-$ original children (39 boys and 23 girls) from the Northern Territory of Australia. The children, who were between 7 and 9 years of age at the start of the study, were measured once each year for 10 years. The children belong to a community that was undergoing a transition from traditional lifestyle to settlement conditions. Relative high infant mortality ( $25 \%$ of live births), high childhood morbidity, and poor growth in the first 2 years of life characterize this sample (Brown and Townsend, 1982).

Finally, the African sample is of children and young adults (55 boys and 62 girls) measured between the ages of 5 and 25 years, living in two neighboring rural villages in Gambia. High rates of disease, seasonal undernutrition, and infant mortality are common in these villages. The poor standard of living is reflected in child growth which, compared with British data, shows "... substantial deficits in both height and weight that develop in early life and which appear to persist without rectification into adulthood" (Billewicz and McGregor, 1982).

\section{Curve fitting}

The Guatemalan data were analyzed using the non-linear optimization program developed by Brown (1983) for fitting the Preece-Baines model I function on an Apple II computer. This version of the function was used since it is the only published, publicly available program of the PreeceBaines model. Moreover, its development for the microcomputer makes its application possible in a wide range of research environments. The Apple II version fits the Preece-Baines function to the longitudinal measurements of an individual through a series of iterations, each iteration minimizing the residual error of the mathematical parameters.

In the present analysis, 400 iterations for 
each child were run. The average value $( \pm \mathrm{SD})$ of the mean error sum of squares is 1.22 (1.59) for boys and 1.44 (1.79) for girls. These values are larger than those reported for studies using main frame computer systems (e.g., Hauspie et al., 1980a; Preece and Baines, 1978), but still relatively small. A Runs Test revealed no significant pattern in the distribution of the residual errors from the fitted curve.

Once the mathematical parameters are calculated, the Apple II program can be used to estimate a series of biological parameters, such as age at "take-off" (the start of the adolescent growth spurt), growth velocity at take-off, age at peak height velocity during the spurt, the value of the peak height velocity, and other adolescent growth events.

The mean values for the mathematical and biological parameters were calculated separately for boys and girls. These means were used to construct mean-constant distance and velocity curves of growth in height. The Apple II program estimates these curves from the average mathematical parameters. Since the biological parameters for the other samples reported in the literature were calculated by different algorithms and different computers, some bias may confound the comparison of results between studies. To allow for direct comparison between all of the samples of children, the mean (or median for Belgians) mathematical parameters published for each of the samples were used to recalculate the mean biological parameters, and the mean-constant curves of growth, using the algorithms of the Apple II program.

\section{RESULTS \\ Mean parameters of growth}

Mathematical and biological parameters of the Preece-Baines model I function for Guatemalan boys are presented in Table 1 . Also presented in Table 1 are the mean values of the parameters for the other six samples of boys. Table 2 contains similar categories of data for Guatemalan girls and the other samples of girls. In both tables the Guatemalan and British data are compared statistically. The British data were chosen for comparison because these were the first data to be described with the Preece-Baines function and have served as the sample for comparison in subsequent studies (e.g., Hauspie et al., 1980b; Brown and Townsend, 1982; Satyanarayana et al., 1989). The Student's t-test was used to contrast each pair of mathematical or biological parameters (standard deviations for the British data published by Preece and Baines [1978] were used to compute these t statistics). Significant contrasts for the boys are the mathematical parameters $\mathrm{P} 1$, which correlates with adult height and several biological parameters, all relating to the adolescent growth spurt. Both the increment in height from take-off (TO) to peak height velocity (PHV) and the increment in height velocity from TO to PHV of the Guatemalan boys is greater than for the British boys. The velocity of growth at PHV is greater for the Guatemalan boys, as is their percentage of adult height achieved at TO and PHV. In contrast, the Guatemalan boys have an earlier age at $\mathrm{TO}$, and a smaller percentage of adult height at TO, than the British boys.

There are no significant differences between the Guatemalan and British girls for any of the parameters.

The mean parameters of growth for the other samples may be compared qualitatively for their similarities and differences with the Guatemalan sample. Belgians (boys and girls) are smaller than Guatemalans in height at TO and height at PHV, but have a larger height increment from PHV to adulthood, and both groups achieve nearly the same adult height (parameter P1). Urban Indians, rural Indians, Australians, and Africans have, generally, smaller statures at TO, PHV, and adulthood and smaller height increments between TO to PHV and PHV to adulthood than Guatemalans. The rural Indian boys, and the Australians of both sexes, have noticeably later ages at $\mathrm{TO}$ and $\mathrm{PHV}$ than do any of the other samples.

\section{Population differences in the velocity and timing of growth events}

These patterns of growth, for the mean parameters of each sample, are displayed graphically in Figures 1 through 4 . The mean-constant velocity curves of growth for Guatemalan, British, and Belgian boys (Fig. 1) show the later age at $T O$ for the British sample and the greater PHV for the Guatemalan sample. Belgians have the smallest amplitude (PHV) of the growth spurt, but continue growing for the longest amount of time after PHV. Guatemalan, British, and Belgian girls (Fig. 2) differ relatively less than do their male counterparts. However, the Guatemalan girls have the latest age at TO and the British and Guatemalan girls have a PHV greater than that of the Belgian 


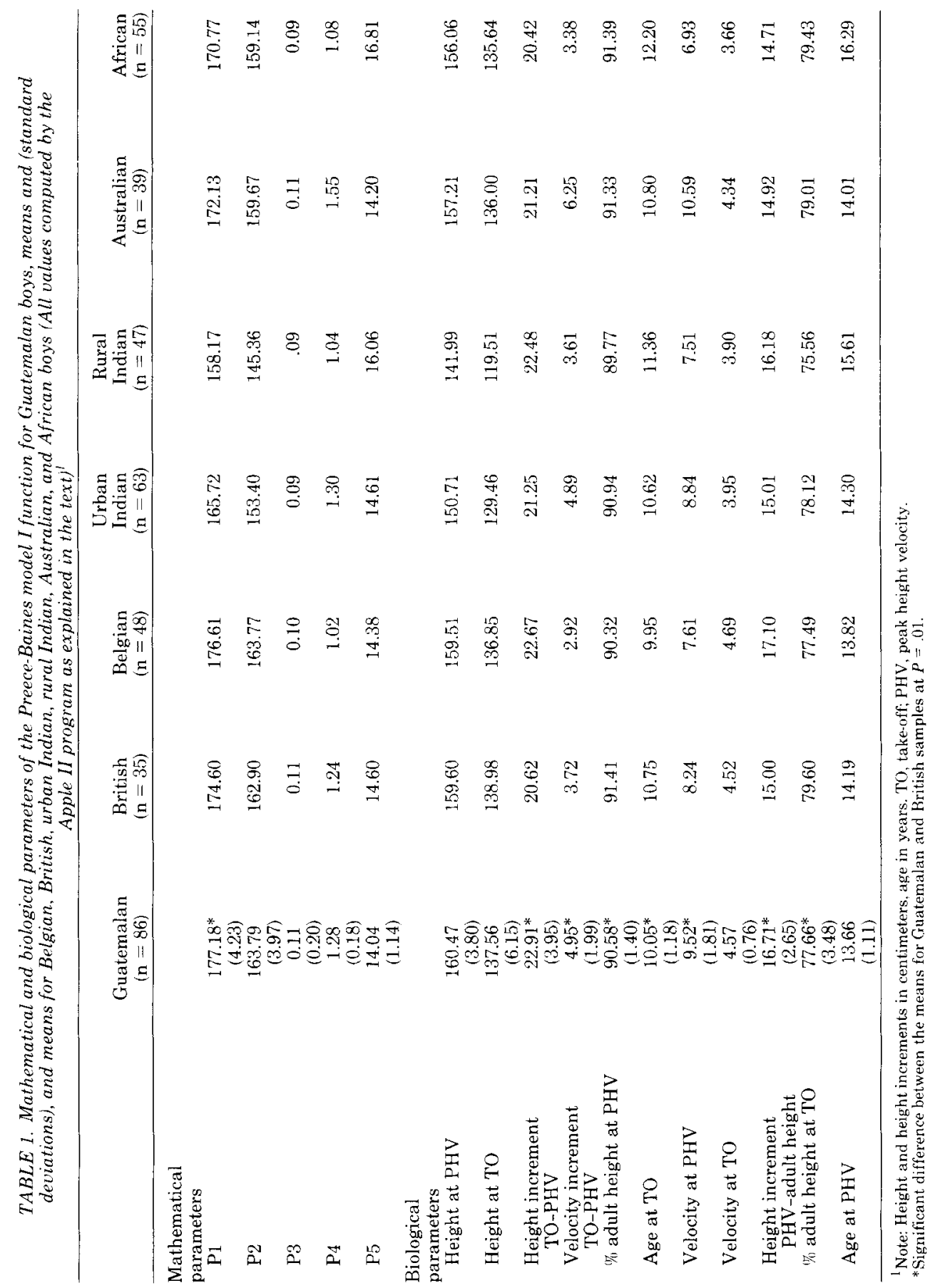




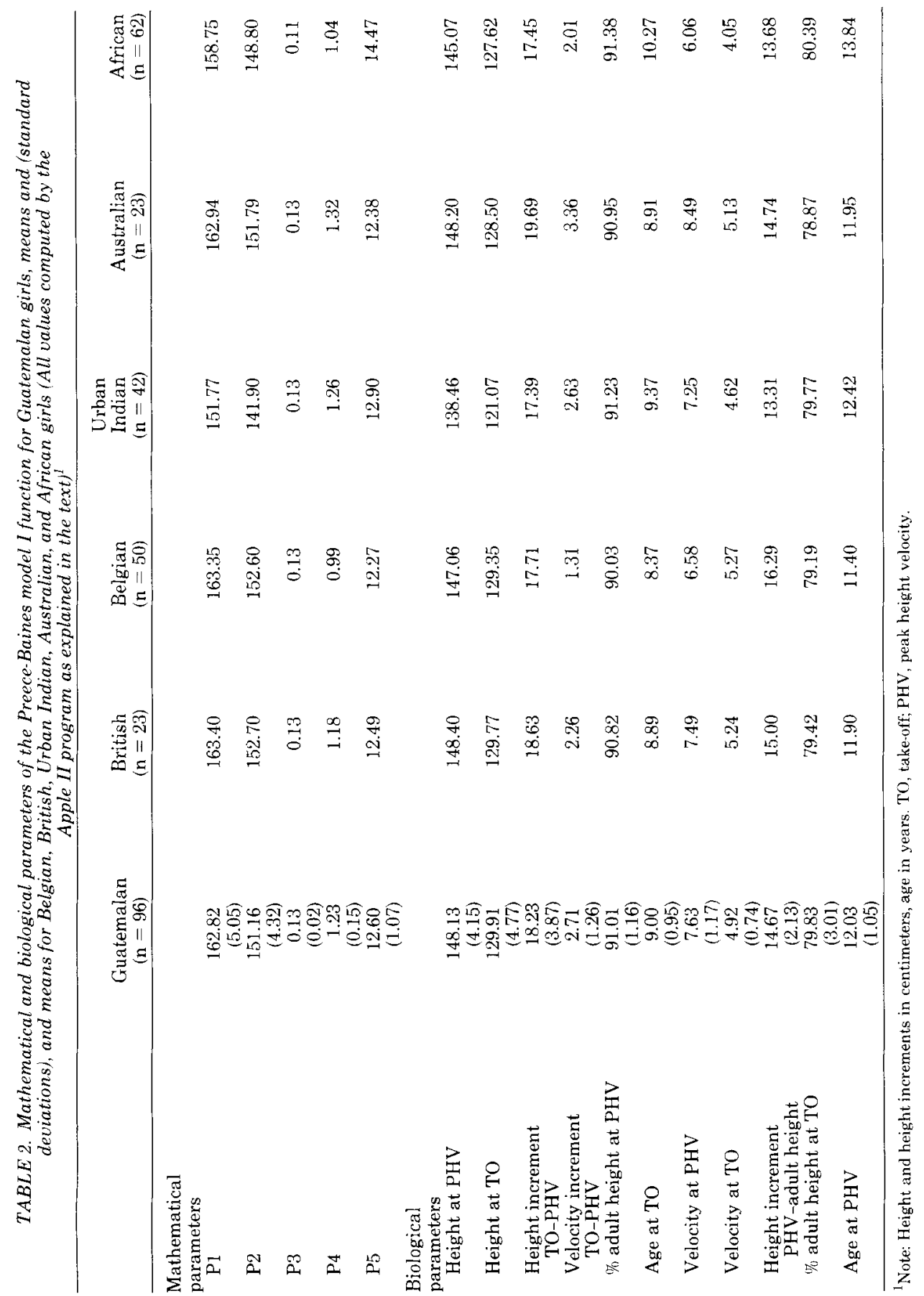




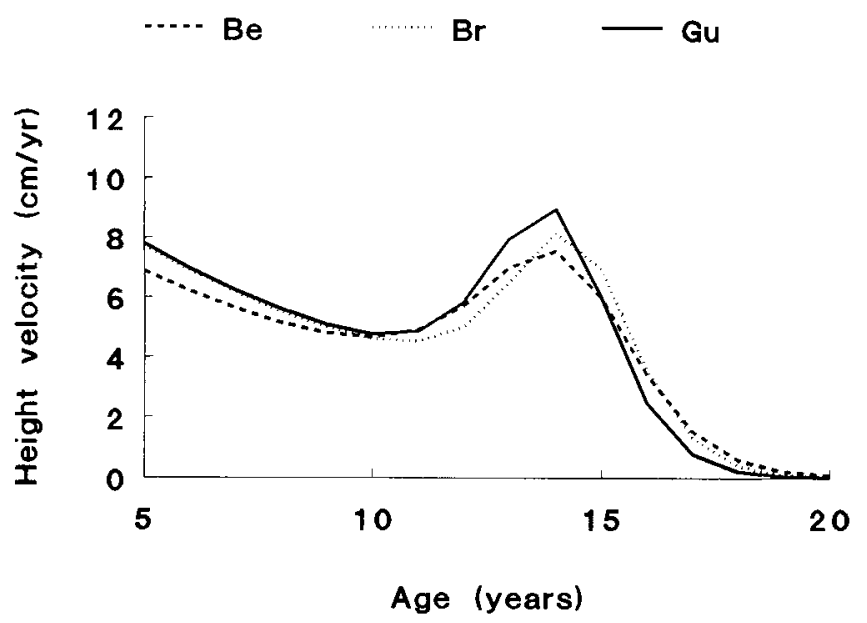

Fig. 1. Height velocity by age for Belgian, British, and Guatemalan boys ( $\mathrm{Be}=$ Belgian; $\mathrm{Br}=$ British; $\mathrm{Gu}=$ Guatemalan).

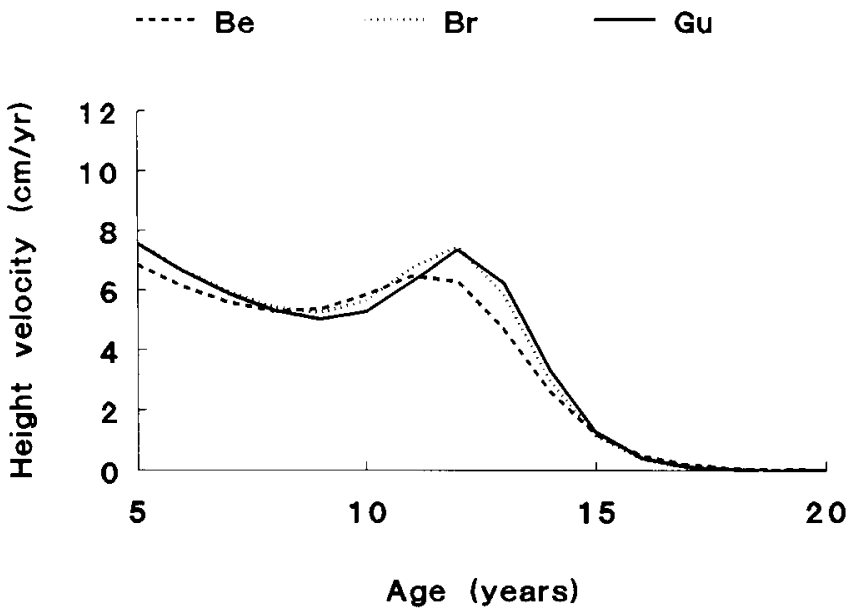

Fig. 2. Height velocity by age for Belgian, British, and Guatemalan girls ( $\mathrm{Be}=$ Belgian; $\mathrm{Br}=$ British; $\mathrm{Gu}=$ Guatemalan).

girls. Despite these differences in patterns of growth, all three groups of girls achieve similar estimated mean adult heights.

In Figure 3 the mean-constant growth velocities for boys from the Guatemalan sample are compared with mean-constant velocities of the urban Indian, rural Indian, Australian, and African samples of boys. In Figure 4 the mean-constant velocities for girls from the urban Indian, Australian, and African samples are compared with those of Guatemalan girls.

Although the timing of growth events (e.g., $\mathrm{TO}$ and $\mathrm{PHV}$ ) of the urban Indian sample is not much different than that for the Guatemalans, urban Indian growth velocity is smaller at all ages. This combination of timing and velocity results in the urban Indians, 

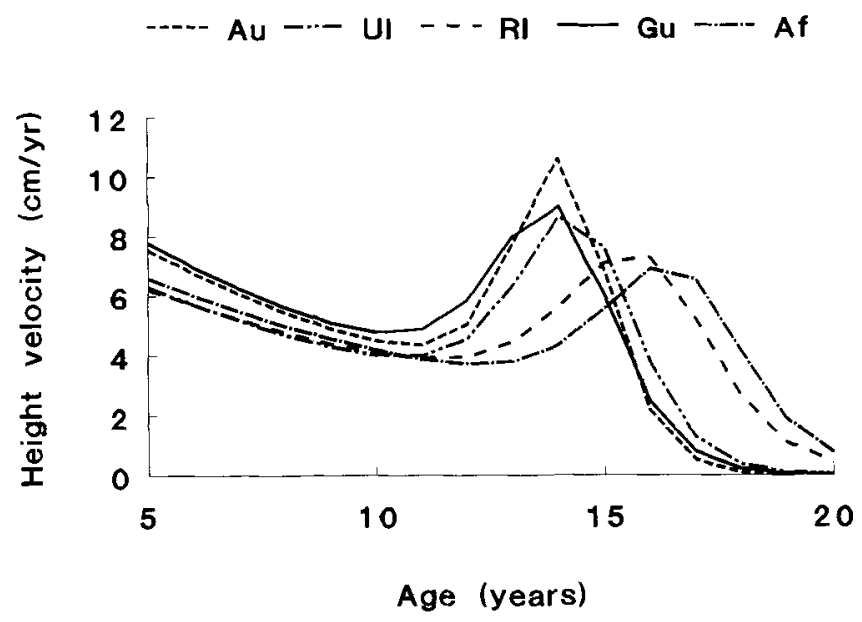

Fig. 3. Height velocity by age for Australian, urban Indian, rural Indian, Guatemalan, and African boys $(\mathrm{Au}=\mathrm{Australian}$; UI=urban Indian; $\mathrm{RI}=$ rural Indian; Gu=Guatemalan; $\mathrm{Af}=$ African).

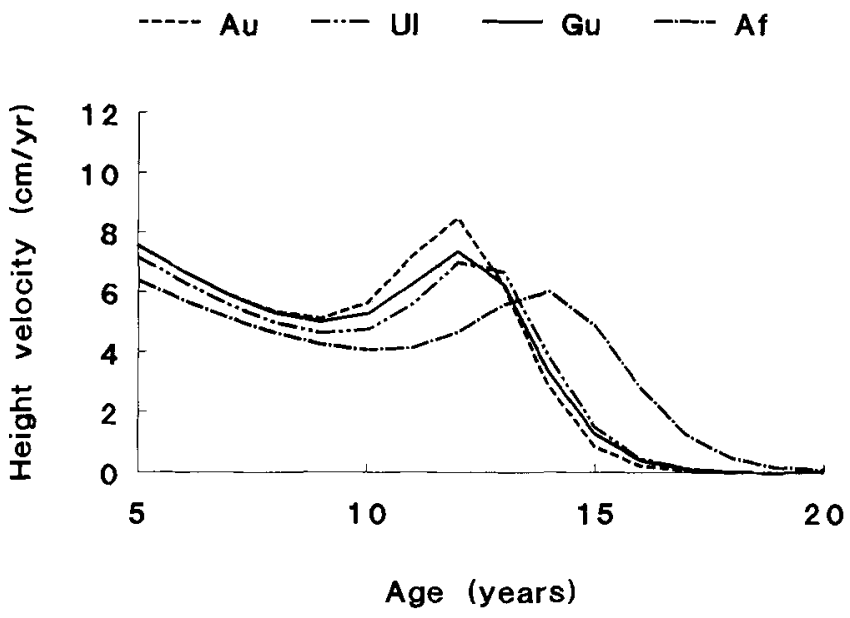

Fig. 4. Height velocity by age for Australian, urban Indian, Guatemalan, and African girls ( $\mathrm{Au}=$ =Australian; $\mathrm{UI}=$ urban Indian; $\mathrm{Gu}=$ Guatemalan; $\mathrm{Af}=$ African).

both boys and girls, having relatively small estimated adult heights. For rural Indian boys and African boys and girls, the age at TO is delayed relative to all the other groups. Likewise, the PHV of the rural Indians and Africans is considerably smaller than that of the other samples. Despite their smaller velocity of growth during childhood and, especially, at adolescence, the rural Indians and
Africans have a longer period of childhood and adolescent growth (note that the PreeceBaines function estimates that growth continues beyond age 20 years). The prolongation of the growing period compensates somewhat for the reduced velocity in growth, and results in an estimated mean adult height for the African men and women that is greater than the estimated mean adult 
height of the urban Indians. However, the prolongation of childhood and adolescent growth of the rural Indian boys does not compensate for low growth velocities, and as adults the rural Indian men do not appear to catch up in size to the urban Indian men.

The velocity curves for the Australian boys and girls have an unusually large amplitude for the adolescent growth spurt. The large PHV is likely to be a real phenomenon since it is noted in graphic analysis of the actual height measurements (as well as in fitted curves such as the Preece-Baines function) and in several studies of Australian Aboriginal growth (Brown and Townsend, 1982). Height velocity during childhood for both Australians and Guatemalans appears to be similar. However, the Preece-Baines estimates for the Australian early childhood velocities (e.g., up to age 7) may be incorrect since the growth data for the Australians begin at age 7 years. Brown and Townsend (1982) report that growth during infancy and early childhood is delayed in this sample of Australians, and suggest that the rapid acceleration in growth that occurs in late childhood and adolescence may represent a "catch-up" in velocity following the early period of growth retardation.

\section{Population variation in sexual dimorphism}

Table 3 presents the differences between men and women in estimated adult height for the six samples that include both boys and girls. The table also gives three components of the total difference in adult sexual dimorphism in height: 1) the sex difference in "adolescent gain," 2) the sex difference in "height at girls' age of TO," and 3) the sex difference in height "due to boys' delay in spurt" (i.e., the boys' longer period of childhood growth prior to take-off).
Guatemalans and urban Indians have the largest sexual dimorphism in adult height. For the Guatemalans, most of this dimorphism is due to the boys' delay in the onset of the spurt and to the difference in adolescent gain. However, for the urban Indians relatively more of the adult sexual dimorphism is due to the difference in height between boys and girls at the girls' age of TO. In this component both the Guatemalans and the urban Indians are different from the other groups, each of which shows a tendency for relatively little sexual dimorphism in height during childhood. The two groups with the greatest sexual dimorphism in adult height also show clear sexual dimorphism at the end of the childhood period of growth. Moreover, the Guatemalans are, on average, the tallest population and the urban Indians are, on average, the shortest population (excluding the rural Indian boys). Sexual dimorphism, therefore, is a phenomenon independent of absolute size.

Differences in the relative contribution of each component to sexual dimorphism in adult height cannot be ascribed simply to overall differences in living conditions. Africans and Australians, who share generally adverse environments for growth, show unique contributions of each component toward final dimorphism. Another anomaly is the negative value of the contribution of "height at girls' age at TO" in both Belgians and Australians. In contrast to the other samples, boys in these two groups are, on average, somewhat shorter than girls at the end of the childhood period of growth.

\section{Correlations between biological parameters}

Correlations between the biological parameters of the Guatemalan samples are presented in Table 4 . The mean values of the biological parameters are useful for describ-

TABLE 3. Differences between men and women in estimated adult height and growth during adolescence for all samples of children (All differences calculated as male value minus female value)

\begin{tabular}{|c|c|c|c|c|}
\hline \multirow[b]{2}{*}{ Samples } & \multicolumn{4}{|c|}{ Differences $(\mathrm{cm})$} \\
\hline & $\begin{array}{l}\text { Adult } \\
\text { height }\end{array}$ & $\begin{array}{l}\text { Adolescent } \\
\text { gain }\end{array}$ & $\begin{array}{c}\text { Height at girls' } \\
\text { age at ' } \mathrm{TO}\end{array}$ & $\begin{array}{l}\text { Due to boys' } \\
\text { delay in spurt }\end{array}$ \\
\hline Guatemalan & 14.36 & 6.71 & 1.28 & 6.87 \\
\hline British & 11.20 & 1.99 & 0.84 & 8.37 \\
\hline Belgian & 13.26 & 5.76 & -0.16 & 7.66 \\
\hline Urban Indian & 13.95 & 5.56 & 3.63 & 4.76 \\
\hline Australian & 9.19 & 1.69 & -1.16 & 8.66 \\
\hline African & 12.02 & 4.00 & 0.21 & 7.81 \\
\hline
\end{tabular}


TABLE 4. Correlation between biological parameters for Guatemalans: Coefficients for boys are above the diagonal and coefficients for girls are below the diagonal

\begin{tabular}{lccccccc} 
& $(1)$ & $(2)$ & $(3)$ & $(4)$ & $(5)$ & $(6)$ & $(7)$ \\
\hline (1) Age at TO & - & $0.85^{*}$ & $0.32^{*}$ & 0.12 & 0.02 & $-0.35^{*}$ & -0.16 \\
(2) Age at PHV & $0.84^{*}$ & - & 0.19 & 0.20 & $-0.37^{*}$ & -0.26 & 0.11 \\
(3) Height at TO & 0.27 & -0.02 & - & $0.78^{*}$ & -0.14 & $-0.65^{*}$ & 0.25 \\
(4) Height at PHV & -0.08 & -0.06 & $0.63^{*}$ & - & -0.13 & -0.31 & $0.79^{*}$ \\
(5) Velocity at TO & $-0.49^{*}$ & $-0.72^{*}$ & 0.11 & 0.08 & - & -0.04 & -0.06 \\
(6) Velocity at PHV & $-0.54^{*}$ & -0.21 & $-0.54^{*}$ & 0.14 & 0.19 & - & 0.06 \\
(7) Parameter P1 & -0.19 & 0.02 & $0.39^{*}$ & $0.91^{*}$ & 0.21 & 0.30 & - \\
\hline
\end{tabular}

*Denotes significance at $P=0.01$.

ing central tendencies within samples and for population comparisons. The analysis of correlations is better suited for understanding the patterns of growth of individual boys and girls within the sample. Both boys and girls have significant positive correlations between age at TO and age at PHV, height at TO and height at PHV, and height at PHV and parameter P1 (estimated adult height). These relationships indicate that during the adolescent period of growth individual children are consistent in their rate of maturation and in size, a finding common to other populations (Hauspie, 1980; Brown and Townsend, 1982; Bogin, 1988, p. 35). Both boys and girls show significant negative correlations between age at $\mathrm{TO}$ and velocity at PHV, height at TO and velocity at PHV, and velocity at TO and age at PHV. These correlations are also common to many other populations (Bogin, 1988).

The Guatemalan children show some differences, by sex, for the correlations between biological parameters. Girls, but not boys, have a significant negative correlation between age at $\mathrm{TO}$ and velocity at $\mathrm{TO}$, and a significant positive correlation between height at TO and parameter P1. Boys, but not girls, have a significant positive correlation between age at TO and height at TO. The causes of the differences between boys and girls for these correlations are not known.

\section{DISCUSSION \\ Curve fitting}

The use of the Preece-Baines model I, adapted for the Apple II microcomputer, provides a useful means for the analysis of longitudinal growth data. Both new data for the Guatemalan sample and published data for the other six samples were analyzed and compared efficiently. Of course, the Apple II microcomputer is not a replacement for mainframe systems or larger and faster microcomputers. Indeed the Apple II program produces results that differ from those obtained with mainframe systems. Primarily, the differences are for the "robustness" of the program to fit the data adequately and the residual error estimates between the original data and the fitted curve. Dr. Hauspie, of the Free University of Brussels, kindly applied his mainframe version of the PreeceBaines model I program to ten cases from the Guatemalan sample. The mainframe program was not able to produce acceptable fitted curves for four of the ten cases. The mean residual sum of squares for the six fitted cases was $0.37( \pm 0.33)$ compared with $0.78( \pm 0.90)$ for the ten cases fitted by the Apple II program (significantly different at $P=.03)$. There were no significant differences between mainframe or microcomputer estimates of the mathematical or biological parameters. Thus, the mainframe program produced a better fit, in the sense that the residual errors were smaller, but both programs produced virtually identical parameter estimates. Moreover, the Apple II program was able to fit adequately all ten cases of longitudinal data.

Another way to evaluate the usefulness of the Apple II program is to compare the mean biological parameters for the Australian sample computed by a mainframe system, published by Brown and Townsend (1982), with the mean biological parameters for the Australians from Tables 1 and 2 of the present report. Since Brown (1983) developed and published the Apple II program based on his experience with the PreeceBaines function on a mainframe, this comparison may be the best "acid test." The Apple II program overestimates the values for some parameters and underestimates 
the value of other parameters. However, the differences between mainframe and microcomputer estimates are small and most would be undetectable if the values were rounded to the nearest whole number.

It is important to state that the PreeceBaines model I function is an abstraction; that is, the model is a numerical formula for forcing a particular mathematical curve to fit a set of data points. The model curve is not the real pattern of growth of the individual child or sample of children; actual growth measurements define the real pattern of growth. Thus, differences between mainframe and microcomputer applications of the model may be more a product of hardware and software configurations than "right" or "wrong" approaches to growth modeling. Given the results of the present analysis, and the comparisons of these results to those for the mainframe, we agree with Brown's (1983) statement that the microcomputer program provides estimates of the parameters that are acceptable for both educational and research purposes. ${ }^{1}$

\section{Population comparisons}

One major benefit of the mathematical modeling of growth is that it allows for precise, quantifiable comparisons between populations. This is of great importance to human biologists when testing hypotheses about the determinants of population variation in amount and rate of growth. Mathematical precision also enables public health workers and clinicians to evaluate the consequences of programs and treatments designed to improve human development.

Previous work by Brown and Townsend (1982) and Satyanarayana et al. (1989) compared mean parameters of the PreeceBaines function for the Australian, British, urban Indian, rural Indian, and African samples. The present analysis extends the comparison of parameters to Belgian and Guatemalan samples. Comparison of the fitted curves shows that there are several paths that may be taken in growth from childhood to adolescence. The Guatemalans, Belgians, British, and urban Indian samples, all from "better" environments for physical development within their nations, follow

\footnotetext{
${ }^{1}$ A translation of the Apple Il program to the MS-DOS computer environment (e.g., IBM compatible machines) should be available by the time this paper is published. Inquiries may be addressed to Dr. Bogin.
}

similar patterns of growth in terms of the timing of adolescent growth events, although they differ significantly in amounts and rates of growth. The only consistent difference between these four groups is that the Guatemalans have a larger peak height velocity (PHV) than the other samples. One may speculate that the differences in stature between these groups have some genetic cause.

The rural Indian, Australian, and African samples live under adverse environmental conditions for human development. Their mean parameters reflect this in the smaller velocities of childhood growth, smaller PHVs (except for the Australian's PHV), and smaller heights achieved at take-off, PHV, and adulthood compared with the Guatemalans, British, and Belgians. Despite the adolescent "catch-up" in growth velocity of the Australians, they remain significantly shorter than Guatemalans as adults. Australians do not differ significantly from British in mean adult height (Brown and Townsend, 1982), and it is estimated that the Australians end up taller than urban Indians as adults. Perhaps the combination of traditional lifestyle and "welfare state" conditions under which this sample of Australian Aborigines lived failed to provide a favorable environment for growth to infants and young children (Brown and Townsend report high morbidity and mortality in the birth to five year old age group), but did meet the growth needs of older children and adolescents.

Rural Indians and Africans appear to have lived under continuously poor environmental conditions for growth. The authors of the original reports for these two samples note clear evidence of chronic undernutrition and high morbidity. Malnutrition and disease are major causes of growth failure. Although both the rural Indian and African children grow for a longer period of time during adolescence, they never recoup the significant difference in height that developed during childhood between them and the Guatemalan, Belgian, and British samples.

Another indication of the impact of malnutrition and disease on growth may be seen in the correlations between the biological parameters of the Preece-Baines model. It is well known that the early maturing child tends to have a more intense adolescent growth spurt, but of shorter duration, than the late maturing child (Boas, 1930; Largo et al., 1978). Hauspie (1980) found that 
across populations this tendency varies widely $(r=-0.05$ to -0.75$)$. For Guatemalan, American, British, Swedish, and Swiss children, the correlations fall toward the high end of this range (Hauspie, 1980; Bogin, 1988 , p. 35), while for Australians (Brown and Townsend, 1982), urban Indian (Hauspie, 1980) and African (Billewicz and McGregor, 1982) the correlations are smaller, though still negative, and not always significant statistically. One interpretation of these findings is that in healthy and wellnourished populations the endogenous biological regulation of the timing and rate of growth is more strongly expressed than in populations suffering from a greater degree of undernutrition and disease, both of which impose limitations on growth.

The analysis of the Guatemalan growth data and their comparison with those of other samples of children and adolescents from around the world show that there are several alternate paths of development that may be taken from childhood to adulthood. Differences in amount of growth, rate of growth, and the timing of adolescent growth events define these paths. These alternate growth trajectories, which are clearly correlated with the nutritional and health environment, are major determinants of population differences in adult size.

\section{ACKNOWLEDGMENTS}

The authors thank Dr. Roland Hauspie for providing unpublished data, for performing computer analyses, and for critical comments that have been incorporated in this paper. We thank Prof. Tasman Brown for sending a copy of his Apple II Preece-Baines function program and for reading a draft of this manuscript. We are grateful to Prof. Frank Johnston, Prof. Gabriel W. Lasker, and two anonymous reviewers for comments that improved the presentation of this paper.

\section{LITERATURE CITED}

Billewicz WZ, and McGregor IA (1982) A birth to maturity longitudinal study of heights and weights in two West African (Gambian) villages. Ann. Hum. Biol. 9:309-320.
Boas F (1930) Observations on the growth of children. Science 72:44-48

Bogin B (1988) Patterns of Human Growth. Cambridge: Cambridge University Press.

Bogin B, and MacVean RB (1978) Growth in height and weight of urban Guatemalan primary school children of high and low socioeconomic elass. Human Biol. 50:477-488.

Bogin B, and MacVean RB (1982) Ethnic and secular influences on the size and maturity of seven year old children living in Guatemala City. Am. J. Phys. Anthropol. 59:393-398.

Bogin B, and MacVean RB (1983) The relationship of socioeconomic status and sex to body size, skeletal maturation, and cognitive status of Guatemala city school children. Child Dev. 54:115-128.

Brown T (1983) The Preece-Baines growth function demonstrated by personal computer: A teaching and research aid. Ann. Hum. Biol. 10:487-489.

Brown T, and Townsend GC (1982) Adolescent growth in height of Australian Aboriginals analysed by the Preece-Baines function: A longitudinal study. Ann. Hum. Biol. 9:495-505.

Cameron N (1986) The methods of auxological anthropology. In Falkner F and Tanner JM (eds.): Human Growth, Vol. 3, Second Edition. New York: Plenum, pp. 3-46.

Hauspie RC (1980) Adolescent growth. In Johnston FE, Roche AF, and Susanne C (eds.): Human Physical Growth and Maturation. New York: Plenum, pp. 161-175.

Hauspie RC, and Wachholder A (1986) Clinical standards for growth velocity in height of Belgian boys and girls, aged 2 to 18. Int. J. Anthropol. 1:339-348.

Hauspie RC, Wachholder A, Baron G, Cantrine F, Susanne C, and Gaffar, M (1980a) A comparative study of the fit of four different functions to the longitudinal growth in height of Belgian girls. Ann. Hum. Biol. $7: 347-358$

Hauspie RC, Das SR, Preece MA, and Tanner JM (1980b) A longitudinal study of the growth in height of boys and girls of West Bengal (India) aged six months to 20 years. Ann. Hum. Biol. 7:429-441.

Johnston FE, Borden M, and MacVean RB (1973) Height, weight and their growth velocities in Guatemalan private schoolchildren of high socioeconomic class. Human Biol. 45:627-641.

Largo RH, Gasser Th, Prader A, Stutzle W, and Huber PJ (1978) Analysis of the adolescent growth spurt using smoothing spline functions. Ann. Hum. Biol. 5:421-434.

Preece MA, and Baines MJ (1978) A new family of mathematical models describing the human growth curve. Ann. Hum. Biol. 5:1-24.

Satyanarayana K, Radhaiah G, Murali Mohan KR, Thimmayamma BVS, Pralhad Rao N, and Narasinga Rao BS (1989) The adolescent growth spurt of height among rural Indian boys in relation to childhood nutritional background: Ân 18 year longitudinal study. Ann. Hum. Biol. 16:289-300.

Wachholder A, and Hauspie RC (1986) Clinical standards for growth in height of Belgian boys and girls aged 2 to 18 years. Int. J. Anthropol. 1:327-338. 